\title{
Exact Solutions of Fragmentation Equations with General Fragmentation Rates and Separable Particles Distribution Kernels
}

\author{
S. C. Oukouomi Noutchie ${ }^{1}$ and E. F. Doungmo Goufo ${ }^{1,2}$ \\ ${ }^{1}$ MaSIM Focus Area, North-West University, Mafikeng 2735, South Africa \\ ${ }^{2}$ Department of Mathematical Sciences, University of South Africa, Florida 1709, South Africa \\ Correspondence should be addressed to S. C. Oukouomi Noutchie; 23238917@nwu.ac.za
}

Received 27 May 2014; Revised 21 June 2014; Accepted 22 June 2014; Published 8 July 2014

Academic Editor: Abdon Atangana

Copyright (c) 2014 S. C. Oukouomi Noutchie and E.F. Doungmo Goufo. This is an open access article distributed under the Creative Commons Attribution License, which permits unrestricted use, distribution, and reproduction in any medium, provided the original work is properly cited.

We make use of Laplace transform techniques and the method of characteristics to solve fragmentation equations explicitly. Our result is a breakthrough in the analysis of pure fragmentation equations as this is the first instance where an exact solution is provided for the fragmentation evolution equation with general fragmentation rates. This paper is the key for resolving most of the open problems in fragmentation theory including "shattering" and the sudden appearance of infinitely many particles in some systems with initial finite particles number.

\section{Preliminaries}

Fragmentation models occur in a large variety of situations including the study of stellar fragments in astrophysics, rock fracture, polymer degradation, DNA fragmentation, aggregates breakage, breakup of solid drugs in organisms, and liquid droplet decay. The theoretical framework of fragmentation dynamics can be traced back to papers by Melzak [1] (analytically) and Filippov [2] (probabilistically). In the 1980s, a systematic investigation of fragmentation models was undertaken, mainly by Ziff and his students, for example $[3,4]$. They provided analytical solutions to a large class of equations of the form

$$
\begin{array}{r}
\frac{\partial}{\partial t} u(t, x)=-a(x) u(t, x)+\int_{x}^{\infty} a(y) b(x \mid y) u(t, y) d y \\
x \geq 0, t>0
\end{array}
$$

with power rates $a(x)=x^{\alpha}, \alpha \in(-\infty, \infty)$ and where $b(x \mid y)$ represented the distribution of particle mass $x$ spawned by the breakage of a particle of mass $y>x$. In their setting, $b(x \mid$ $y$ ) was given by a power law

$$
b(x \mid y)=(\nu+2) \frac{x^{\nu}}{y^{\nu+1}},
$$

with $v \in(-2,0$ ] (see also [5] for a more insight regarding this case). Note that the density of particles having mass $x$ at time $t$ is denoted by $u(t, x)$. Additionally in absence of any other mechanism, the mass of all daughter particles is equal to the mass of the parent. This "local" conservation mass principle is given by

$$
\int_{0}^{y} x b(x \mid y) d x=y
$$

In a similar way, the amount of particles created by a particle of size $y$ is given by

$$
n(y)=\int_{0}^{y} b(x \mid y) d x
$$

and $n(y)$ may be infinite. 
Formal conservation principles

$$
\begin{aligned}
\frac{d}{d t} M(t) & =\int_{0}^{\infty} \frac{\partial}{\partial t} u(t, x) x d x=0 \\
\frac{d}{d t} N(t) & =\int_{0}^{\infty} \frac{\partial}{\partial t} u(t, x) d x \\
& =\int_{0}^{\infty} a(x)(n(x)-1) u(t, x) d x
\end{aligned}
$$

can be obtained through the means of local conservation principles (3) and (4) and the integration of (1).

This paper extends the class of power law rates to any positive and continuous function on $(0, \infty)$ and it is assumed that $b$ is separable and can be written as

$$
b(x \mid y)=\beta(x) \gamma(y)
$$

where

$$
\gamma(y)=\frac{y}{\int_{0}^{y} s \beta(s) d s}
$$

in order to satisfy the local mass conservation rule. Moreover, it is assumed that $\beta$ is any continuous function on $(0, \infty)$. A generalization of the power law $b$ described in (2) can be found in (6) which has the advantage of allowing the number of daughter particles,

$$
n(y)=\frac{y \int_{0}^{y} \beta(s) d s}{\int_{0}^{y} s \beta(s) d s}
$$

to depend on the parent size $y[6]$.

Due to the inability of getting exact solutions in fragmentation models, various authors have used several functional analytic approaches to investigate the dynamics of the system. These methods include semigroup theory [7-10], perturbation theory [11-13], approximation techniques [14], and probabilistic methods $[2,15]$. The efficiency of these methods is limited as these problems are reformulated in abstract spaces that are norm dependent and the overall behavior of the dynamics changes radically as different metrics are included in the system. The Laplace transforms as extensively discussed in $[16,17]$ will play a key role in our investigations. Next we introduce a theorem and a definition that are instrumental in the analysis required in the obtention of exact solutions.

Theorem 1 (see [6]). Assume that $\lim _{x \rightarrow 0^{+}} a(x)$ exists (finite or infinite). Then the fragmentation equation 1 is conservative if and only if there exists $\delta>0$ such that $b(x \mid x) / a(x) \notin$ $L_{1}([0, \delta])$.

\subsection{Laplace Transforms}

Definition 2. The Laplace transform of a piecewise continuous function $f(t), 0 \leq t<+\infty$ is the function $F(s)=\mathscr{L}\{f(t)\}$ defined by

$$
F(s)=\int_{0}^{\infty} e^{-s t} f(t) d t
$$

The inverse Laplace transform of $F(s)$ is $f(t), f(t)=$ $\mathscr{L}^{-1}(F(s))$.

\section{Solvability of the Fragmentation Equation}

In this section, we use Laplace transform to solve the fragmentation equation

$$
\begin{gathered}
\frac{\partial}{\partial t} u(t, x)=-a(x) u(t, x)+\int_{x}^{\infty} a(y) b(x \mid y) u(t, y) d y \\
x \geq 0, t>0 \\
u(0, x)=u_{0}(x) .
\end{gathered}
$$

Let $\widetilde{u}(s, x)=\mathscr{L}[u(t, x)]$. Clearly, we have that

$$
\begin{gathered}
\mathscr{L}\left\{\frac{\partial}{\partial t} u(t, x)\right\}=s \tilde{u}(s, x)-u_{0}(x), \\
\mathscr{L}\{a(x) u(t, x)\}=a(x) \tilde{u}(s, x), \\
\mathscr{L}\left\{\int_{x}^{\infty} a(y) b(x \mid y) u(t, y) d y\right\} \\
=\int_{x}^{\infty} a(y) b(x \mid y) \tilde{u}(s, y) d y .
\end{gathered}
$$

Making use of (10) and (11), we obtain the following equation:

$$
\begin{aligned}
s \tilde{u}(s, x)-u_{0}(x)= & -a(x) \tilde{u}(s, x) \\
& +\int_{x}^{\infty} a(y) b(x \mid y) \tilde{u}(s, y) d y
\end{aligned}
$$

that is,

$$
u_{0}(x)=(s+a(x)) \tilde{u}(s, x)-\int_{x}^{\infty} a(y) b(x \mid y) \widetilde{u}(s, y) d y .
$$

Viewing $s$ as a parameter, this is similar to the resolvent equation solved in 2010 (Banasiak and Noutchie). The solution reads as

$$
\begin{aligned}
\tilde{u}(s, x)= & \frac{u_{0}(x)}{s+a(x)}+\frac{\beta(x)}{s+a(x)} e^{-\xi_{s}(x)} \\
& \times \int_{x}^{\infty} \frac{a(y) \gamma(y)}{s+a(y)} e^{\xi_{s}(y)} u_{0}(y) d y,
\end{aligned}
$$

where

$$
\xi_{s}(x)=\int_{1}^{x} \frac{a(\eta) b(\eta \mid \eta)}{s+a(\eta)} d \eta
$$


The solution $u(t, x)$ of $(10)$ is the inverse Laplace transform of $\widetilde{u}(s, x)$. Clearly,

$$
\begin{gathered}
\mathscr{L}^{-1}\left\{\frac{u_{0}(x)}{s+a(x)}\right\}=u_{0}(x) \mathscr{L}^{-1}\left\{\frac{1}{s+a(x)}\right\}=u_{0}(x) e^{-t a(x)}, \\
\mathscr{L}^{-1}\left\{\frac{\beta(x)}{s+a(x)} e^{-\xi_{s}(x)} \int_{x}^{\infty} \frac{a(y) \gamma(y)}{s+a(y)} e^{\xi_{s}(y)} u_{0}(y) d y\right\} \\
=\int_{x}^{\infty} a(y) b(x \mid y) u_{0}(y) \mathscr{L}^{-1} \\
\times\left\{\frac{1}{s+a(x)} \frac{1}{s+a(y)} e^{\left\{\xi_{s}(y)-\xi_{s}(x)\right\}}\right\} d y \\
=\int_{x}^{\infty} a(y) b(x \mid y) u_{0}(y) \mathscr{L}^{-1}\{\Theta(s, x, y)\} d y,
\end{gathered}
$$

where

$\Theta(s, x, y)=\frac{1}{s+a(x)} \frac{1}{s+a(y)} \exp \left\{\int_{x}^{y} \frac{a(\eta) b(\eta \mid \eta)}{s+a(\eta)} d \eta\right\}$.

Therefore, the solution of the fragmentation equation

$$
\begin{array}{r}
\frac{\partial}{\partial t} u(t, x)=-a(x) u(t, x)+\int_{x}^{\infty} a(y) b(x \mid y) u(t, y) d y, \\
x \geq 0, \quad t>0, \\
u(0, x)=u_{0}(x)
\end{array}
$$

is given by

$$
\begin{aligned}
u(t, x)= & u_{0}(x) e^{-t a(x)} \\
& +\int_{x}^{\infty} a(y) b(x \mid y) u_{0}(y) \mathscr{L}^{-1}\{\Theta(s, x, y)\} d y .
\end{aligned}
$$

\section{Applications}

In this section, we assume that

$$
\begin{gathered}
a(x)=x^{\alpha+1}, \quad \alpha \in(-\infty, \infty), \\
b(x \mid y)=(2+\nu) \frac{x^{\nu}}{y^{\nu+1}},
\end{gathered}
$$

with $v \in(-2,0]$. We have

$$
\begin{aligned}
\int_{x}^{y} \frac{a(\eta) b(\eta \mid \eta)}{s+a(\eta)} d \eta & =(2+\nu) \int_{x}^{y} \frac{\eta^{\alpha}}{s+\eta^{\alpha+1}} d \eta \\
& =\frac{2+\nu}{\alpha+1} \ln \left\{\frac{s+y^{\alpha+1}}{s+x^{\alpha+1}}\right\} ;
\end{aligned}
$$

it follows that

$$
\exp \left\{\int_{x}^{y} \frac{a(\eta) b(\eta \mid \eta)}{s+a(\eta)} d \eta\right\}=\left\{\frac{s+y^{\alpha+1}}{s+x^{\alpha+1}}\right\}^{\gamma},
$$

where

$$
\gamma=\frac{2+\nu}{\alpha+1}
$$

Thus

$$
\Theta_{\alpha, \nu}(s, x, y)=\frac{\left(s+y^{\alpha+1}\right)^{\gamma-1}}{\left(s+x^{\alpha+1}\right)^{\gamma+1}}=\left\{\frac{1}{s+x^{\alpha+1}}\right\}^{\gamma+1}\left\{s+y^{\alpha+1}\right\}^{\gamma-1} .
$$

Therefore, the solution $u(t, x)$ is given by

$$
\begin{aligned}
& u(t, x)= u_{0}(x) e^{-t x^{\alpha+1}} \\
&+(2+\nu) \int_{x}^{\infty}\left\{\frac{x}{y}\right\}^{\nu} y^{\alpha} u_{0}(y) \mathscr{L}^{-1} \\
& \times\left\{\Theta_{\alpha, \nu}(s, x, y)\right\} d y .
\end{aligned}
$$

3.1. Case $\alpha=-3$ and $v=0$. We want to solve the following equation:

$$
\begin{gathered}
\frac{\partial}{\partial t} u(t, x)=-x^{-2} u(t, x)+2 \int_{x}^{\infty} y^{-3} u(t, y) d y, \\
u(0, x)=u_{0}(x) .
\end{gathered}
$$

We have $\gamma=-1$; it follows that

$$
\Theta_{-3,0}(s, x, y)=\left\{\frac{1}{s+x^{-2}}\right\}^{0}\left\{s+y^{-2}\right\}^{-2}=\left\{s+y^{-2}\right\}^{-2} .
$$

Thus

$$
\mathscr{L}^{-1}\left\{\Theta_{-3,0}(s, x, y)\right\}=\mathscr{L}^{-1}\left\{\left(s+y^{-2}\right)^{-2}\right\}=t e^{-t y^{-2}} .
$$

Therefore,

$$
u(t, x)=u_{0}(x) e^{-t x^{-2}}+2 t \int_{x}^{\infty} y^{-3} e^{-t y^{-2}} u_{0}(y) d y .
$$

3.2. Case $\alpha=-2$ and $\nu=0$. We want to solve the following equation:

$$
\begin{gathered}
\frac{\partial}{\partial t} u(t, x)=-x^{-1} u(t, x)+2 \int_{x}^{\infty} y^{-2} u(t, y) d y, \\
u(0, x)=u_{0}(x) .
\end{gathered}
$$

We have $\gamma=-2$; it follows that

$$
\begin{aligned}
\Theta_{-2,0}(s, x, y) & =\left\{\frac{1}{s+x^{-1}}\right\}^{-1}\left\{s+y^{-1}\right\}^{-3} \\
& =\frac{s+y^{-1}-y^{-1}+x^{-1}}{\left(s+y^{-1}\right)^{3}} \\
& =\frac{1}{\left(s+y^{-1}\right)^{2}}+\frac{\left(x^{-1}-y^{-1}\right)}{\left(s+y^{-1}\right)^{3}} .
\end{aligned}
$$


Thus

$$
\begin{aligned}
\mathscr{L}^{-1}\left\{\Theta_{-2,0}(s, x, y)\right\}= & \mathscr{L}^{-1}\left\{\frac{1}{\left(s+y^{-1}\right)^{2}}\right\} \\
& +\left(x^{-1}-y^{-1}\right) \mathscr{L}^{-1}\left\{\frac{1}{\left(s+y^{-1}\right)^{3}}\right\} \\
= & t e^{-t y^{-1}}+\left(x^{-1}-y^{-1}\right) e^{-t y^{-1}} \frac{t^{2}}{2} .
\end{aligned}
$$

Therefore,

$$
\begin{aligned}
u(x, t)= & e^{-t / x} u_{0}(x)+2 t \int_{x}^{\infty} \frac{e^{-t / y}}{y^{2}} e^{-t / y} u_{0}(y) d y \\
& +t^{2} \int_{x}^{\infty} \frac{e^{-t / y}}{y^{2}}\left(\frac{1}{x}-\frac{1}{y}\right) u_{0}(y) d y
\end{aligned}
$$

3.3. General Case $a(x)=x^{\alpha+1}$ and $b(x \mid y)=(2+\nu)\left(x^{\nu} / y^{\nu+1}\right)$. We want to solve

$$
\begin{gathered}
\frac{\partial}{\partial t} u(t, x)=-x^{\alpha+1} u(t, x)+(2+v) x^{\nu} \int_{x}^{\infty} y^{\alpha-v} u(t, y) d y \\
x \geq 0, \quad t>0 \\
u(0, x)=u_{0}(x) .
\end{gathered}
$$

From the previous section, the solution of this equation is

$$
\begin{aligned}
u(t, x)= & u_{0}(x) e^{-t x^{\alpha+1}}+(2+v) \\
& \times \int_{x}^{\infty}\left\{\frac{x}{y}\right\}^{v} y^{\alpha} u_{0}(y) \mathscr{L}^{-1}\left\{\Theta_{\alpha, v}(s, x, y)\right\} d y .
\end{aligned}
$$

Note that

$$
\begin{aligned}
\mathscr{L}^{-1}\left\{\Theta_{\alpha, \nu}(s, x, y)\right\}= & \mathscr{L}^{-1}\left\{\left\{\frac{1}{s+x^{\alpha+1}}\right\}^{\gamma+1}\left\{s+y^{\alpha+1}\right\}^{\gamma-1}\right\} \\
\mathscr{L}^{-1}\left\{\Theta_{\alpha, \nu}(s, x, y)\right\}= & \mathscr{L}^{-1}\left\{\left\{\frac{1}{s+x^{\alpha+1}}\right\}^{\gamma+1}\left\{s+y^{\alpha+1}\right\}^{\gamma-1}\right\} \\
= & \operatorname{texp}\left(-t x^{\alpha+1}\right)_{1} F_{1} \\
& \times\left(1-\gamma ; 2 ; t\left(x^{\alpha+1}-y^{\alpha+1}\right)\right) .
\end{aligned}
$$

It follows that

$$
\begin{aligned}
u(t, x)= & u_{0}(x) e^{-t x^{\alpha+1}} \\
& +(2+v) t \exp \left(-t x^{\alpha+1}\right) \\
& \times \int_{x}^{\infty}\left\{\frac{x}{y}\right\}^{\nu}{ }_{1} F_{1}\left(1-\gamma ; 2 ; t\left(x^{\alpha+1}-y^{\alpha+1}\right)\right) \\
& \times y^{\alpha} u_{0}(y) d y .
\end{aligned}
$$

We recover the results of Ziff and his students [3, 4, 18].

\section{Concluding Remarks}

In this paper, we successfully used Laplace transforms and the methods of characteristics to solve an open problem in applied mathematics derived over 60 years ago. We extended the work of Ziff and his students and provided the full solution of fragmentation equations with general explosion rates. This work enables the computation of removal rates and shattering in fragmentation models and provides a general framework for understanding particles distributions in fragmentation processes as time evolves. In particular, it enables a complete classification of shattering regimes.

\section{Conflict of Interests}

The authors declare that there is no conflict of interests regarding the publication of this paper.

\section{References}

[1] Z. A. Melzak, "A scalar transport equation," Transactions of the American Mathematical Society, vol. 85, pp. 547-560, 1957.

[2] I. Filippov, "On the distribution of the sizes of particles which undergo splitting," Theory of Probability and Its Applications, vol. 6, pp. 275-293, 1961.

[3] R. M. Ziff and E. D. McGrady, "Kinetics of polymer degradation," Macromolecules, vol. 19, no. 10, pp. 2513-2519, 1986.

[4] R. M. Ziff and E. D. McGrady, "The kinetics of cluster fragmentation and depolymerisation," Journal of Physics A: Mathematical and General, vol. 18, no. 15, pp. 3027-3037, 1985.

[5] J. Banasiak and S. C. Oukouomi Noutchie, "Controlling number of particles in fragmentation equations," Physica D: Nonlinear Phenomena, vol. 239, no. 15, pp. 1422-1435, 2010.

[6] J. Banasiak, "Conservative and shattering solutions for some classes of fragmentation models," Mathematical Models \& Methods in Applied Sciences, vol. 14, no. 4, pp. 483-501, 2004.

[7] J. Banasiak and L. Arlotti, Perturbations of Positive Semigroups with Applications , Springer Monographs in Mathematics, Springer, London, UK, 2006.

[8] K. Engel and R. Nagel, One-Parameter Semigroups for Linear Evolution Equations, vol. 194 of Graduate Texts in Mathematics, Springer, 2000.

[9] D. J. McLaughlin, W. Lamb, and A. C. McBride, "A semigroup approach to fragmentation models," SIAM Journal on Mathematical Analysis, vol. 28, no. 5, pp. 1158-1172, 1997.

[10] A. Pazy, Semigroups of Linear Operators and Applications to Partial Differential Equations, Applied Mathematical Sciences, 1983.

[11] A. Atangana, "On the singular perturbations for fractional differential equation," The Scientific World Journal, vol. 2014, Article ID 752371, 9 pages, 2014.

[12] S. C. Oukouomi Noutchie, "Analysis of the effects of fragmentation-coagulation in planktology," Comptes RendusBiologies, vol. 333, no. 11-12, pp. 789-792, 2010.

[13] S. C. Oukouomi Noutchie and E. F. Doungmo Goufo, "Global solvability of a continuous model for nonlocal fragmentation dynamics in a moving medium," Mathematical Problems in Engineering, vol. 2013, Article ID 320750, 8 pages, 2013.

[14] S. C. Oukouomi Noutchie, "Existence and uniqueness of conservative solutions for nonlocal fragmentation models," 
Mathematical Methods in the Applied Sciences, vol. 33, no. 15, pp. 1871-1881, 2010.

[15] M. H. Ernst and G. Szamel, "Fragmentation kinetics," Journal of Physics A: Mathematical and General, vol. 26, no. 22, pp. 60856091, 1993.

[16] A. Atangana, "A note on the triple Laplace transform and its applications to some kind of third-order differential equation," Abstract and Applied Analysis, vol. 2013, Article ID 769102, 10 pages, 2013.

[17] A. Atangana and S. C. Oukouomi Noutchie, "On multi-laplace transform for solving nonlinear partial differential equations with mixed derivatives," Mathematical Problems in Engineering, vol. 2014, Article ID 267843, 9 pages, 2014.

[18] R. M. Ziff and E. D. McGrady, "'Shattering' transition in fragmentation," Physical Review Letters, vol. 2, pp. 3027-3037, 1985. 


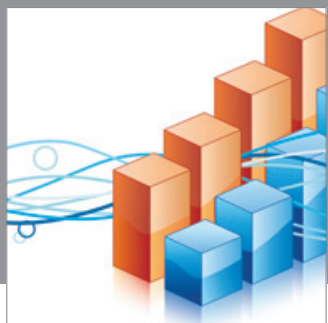

Advances in

Operations Research

mansans

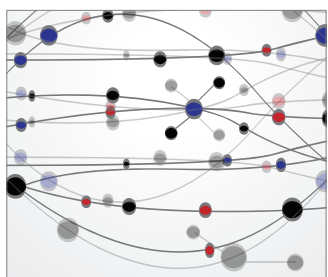

The Scientific World Journal
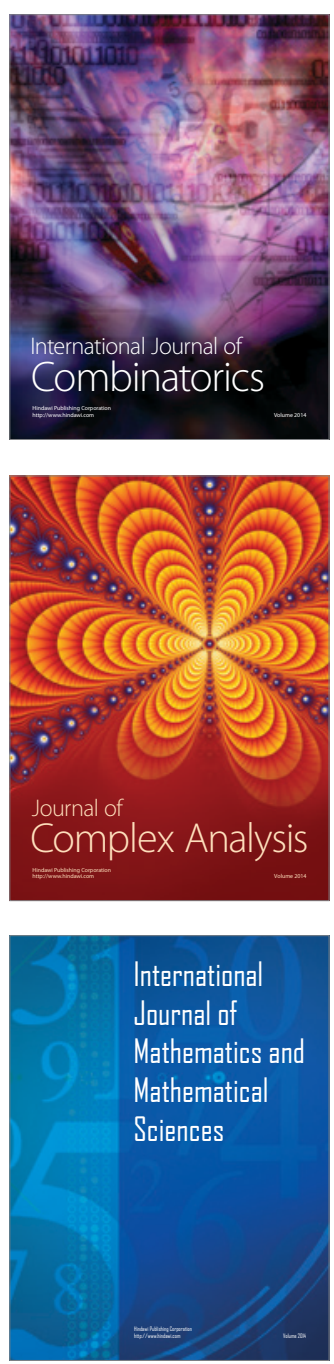
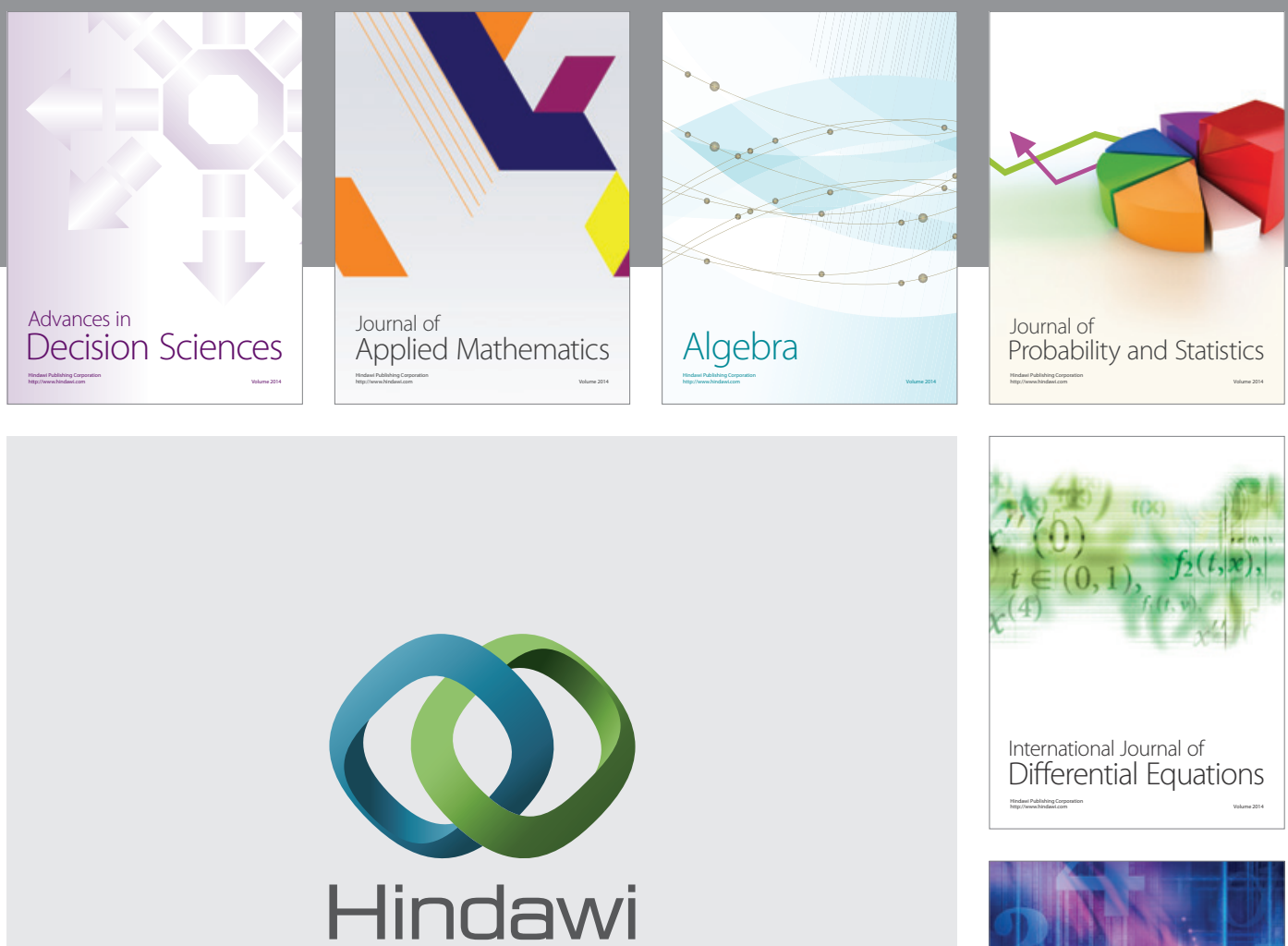

Submit your manuscripts at http://www.hindawi.com
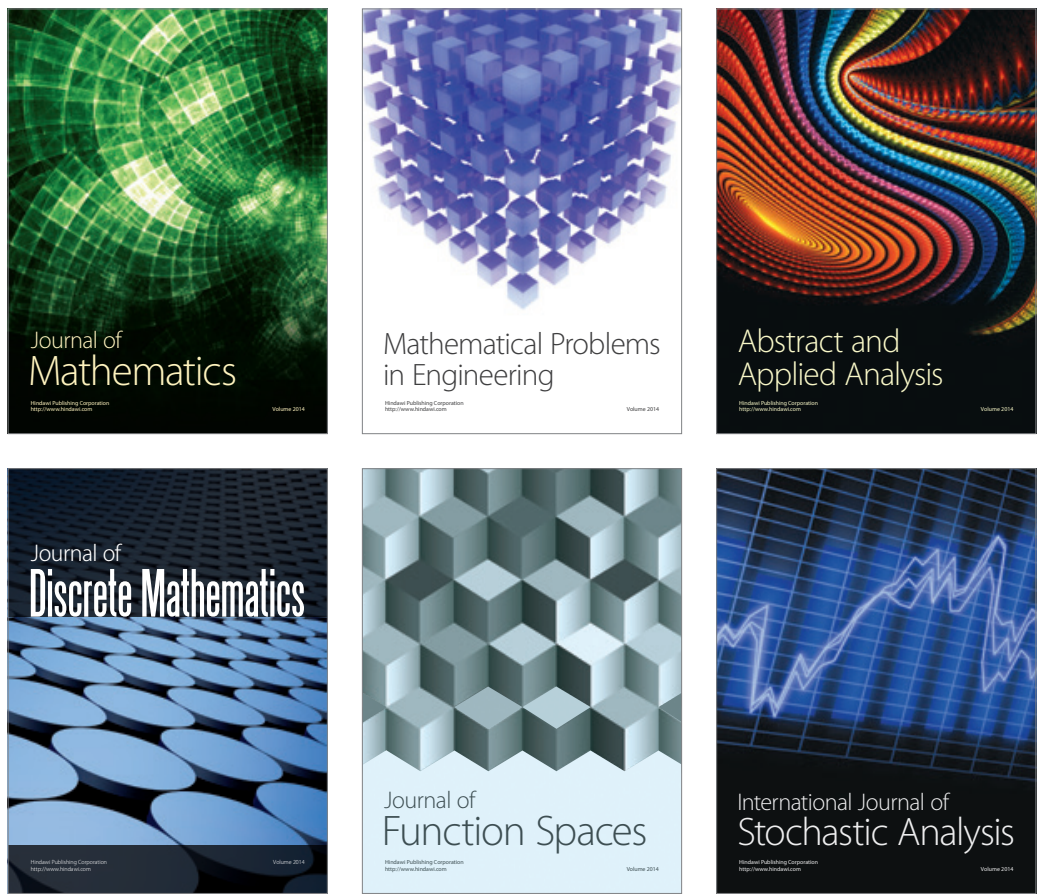

Journal of

Function Spaces

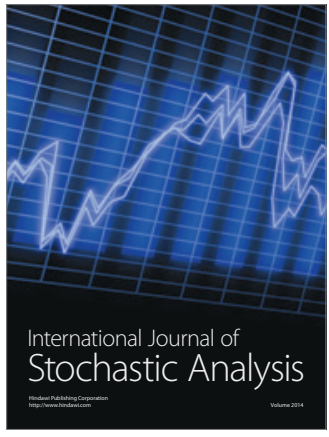

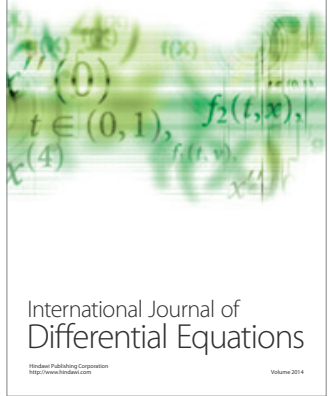
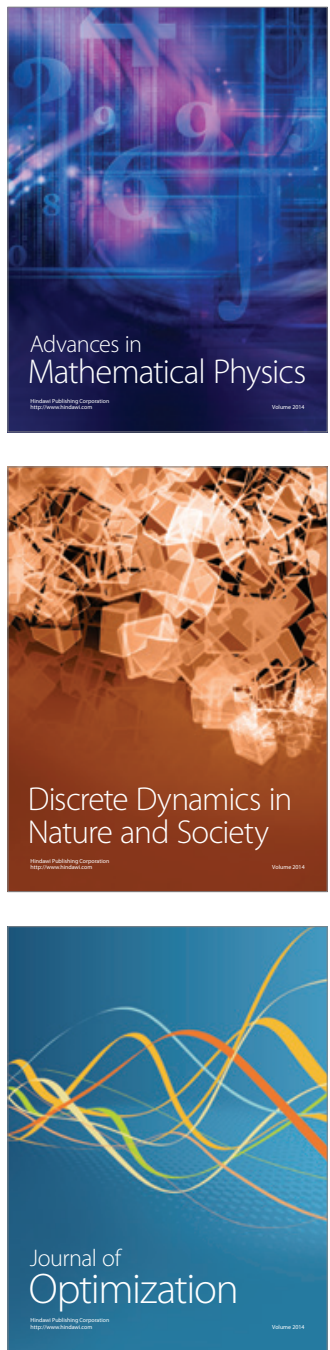